\title{
Enoyl-coenzyme A hydratase short chain 1 silencing attenuates the proliferation of hepatocellular carcinoma by inhibiting epidermal growth factor signaling in vitro and in vivo
}

\author{
BI-YUN LIN ${ }^{1 *}$, CHUAN-XING XIAO ${ }^{2 *}$, WEN-XIU ZHAO ${ }^{1}$, LI XIAO $^{2}$, \\ XU $\mathrm{CHEN}^{2}$, PING $\mathrm{LI}^{2}$ and XIAO-MIN WANG ${ }^{1}$
}

${ }^{1}$ Fujian Provincial Key Laboratory of Chronic Liver Disease and Hepatocellular Carcinoma, Department of Hepatobiliary Surgery;

${ }^{2}$ Department of Gastroenterology, Zhongshan Hospital Affiliated to Xiamen University, Xiamen, Fujian 361004, P.R. China

Received June 17, 2014; Accepted February 17, 2015

DOI: $10.3892 / \mathrm{mmr} .2015 .3453$

\begin{abstract}
Enoyl-coenzyme A hydratase short chain 1 (ECHS1) regulates fatty acid metabolism and is an essential factor in tumor development. The present study aimed to investigate the molecular mechanisms of ECHS1 in hepatocellular carcinogenesis by studying proliferation and survival in ECHS1 knocked-down hepatocellular carcinoma (HCC) cell lines, HepG2 and HuH7. The effect of ECHS1 on tumor development was investigated by tumor transplantation in nude mice, and the signaling pathways involved in the ECHS1-mediated regulation of HCC cell proliferation were identified by western blot analysis. The silencing of ECHS1 suppressed HCC cell proliferation in vitro and suppressed the growth of transplanted tumors in vivo. In addition, the phosphorylation of EGFR and its downstream effectors ERK1/2 and AKT was downregulated in ECHS1 knocked-down cells and tumor tissues. Furthermore, knockdown of ECHS1 in HCC suppressed cyclin D3 and cyclin dependent kinase 6 expression, whilst enhancing p16 and p21 expression. Therefore, ECHS1 may also be involved in cell cycle progression in HCC cells. These results suggested that ECHS1 may promote cell proliferation in HCC in an EGFR-dependent manner.
\end{abstract}

Correspondence to: Professor Xiao-Min Wang, Fujian Provincial Key Laboratory of Chronic Liver Disease and Hepatocellular Carcinoma, Department of Hepatobiliary Surgery, Zhongshan Hospital Affiliated to Xiamen University, 209 South Hubin Road, Xiamen, Fujian 361004, P.R. China

E-mail: wangxiaomin2203@163.com

*Contributed equally

Abbreviations: HCC, hepatocellular carcinoma; ECHS1, enoylcoenzyme A hydratase short chain 1; EGFR, epidermal growth factor receptor; CCK-8, cell counting kit-8

Key words: enoyl-coenzyme A hydratase short chain 1, hepatocellular carcinoma, proliferation, epidermal growth factor receptor signaling

\section{Introduction}

Hepatocellular carcinoma (HCC) is the fifth most common malignant cancer and the third leading cause of cancer-associated mortality worldwide (1). Despite remarkable achievements in improving HCC treatment, patient prognosis generally remains very poor (2). Although numerous molecules and signaling pathways involved in the development of HCC have been identified (3-6), the molecular mechanisms underlying the tumorigenesis and proliferation of $\mathrm{HCC}$ have remained elusive.

Enoyl-coenzyme A (CoA) hydratase short chain 1 (ECHS1) catalyzes the hydration of 2-trans-enoyl-CoA intermediates to form L-3-hydroxyacyl-CoAs, which constitutes the second step in the $\beta$-oxidation pathway of fatty acid metabolism (7). In addition to its role in fatty acid metabolism, ECHS1 may also be involved in tumor development. A proteomics study revealed that ECHS1 was consistently upregulated in prostate cancer tissues and is therefore considered a candidate disease biomarker in prostate needle-biopsy specimens (8). ECHS1 was also identified as a candidate gene in human colorectal carcinogenesis $(9,10)$. Downregulation of ECHS1 enhances PP2-induced apoptosis in human breast cancer MCF-7 cells (11). Chang et al demonstrated that ECHS1 interacts with and inhibits signal transducer and activator of transcription 3 phosphorylation, transcriptional activity and subsequent target gene expression (12). The results of a previous study by our group confirmed ECHS1 as a novel hepatitis B surface antigen (HB) binding protein that enhances HepG2 cell apoptosis by reducing the mitochondrial membrane potential; and explored the potential role of ECHS1 in HCC (13), although the precise molecular mechanisms of ECHS1 in HCC progression remain to be elucidated.

Several studies have demonstrated aberrant activation of epidermal growth factor receptor (EGFR) in HCC pathogenesis (14-17). Significant alterations in the downstream cellular signaling cascades, including phosphoinositide 3 kinase $(\mathrm{PI} 3 \mathrm{~K}) / \mathrm{Akt} / \mathrm{mammalian}$ target of rapamycin (mTOR) and Raf/mitogen activated protein kinase kinase (MEK)/extracellular signal-regulated kinase (ERK), as well 
as altered gene expression result in hepatoma formation via increased proliferation, cell-cycle progression and apoptotic resistance (14,18-20). However, the molecular mechanisms underlying these effects have remained elusive. The present study aimed to investigate the molecular mechanisms of ECHS1 in HCC cells in vitro and in vivo.

\section{Materials and methods}

Ethics statement. The present study was approved by the Ethics Committee of Zhongshan Hospital Affiliated to Xiamen University (no. 20081009; Xiamen, China). All procedures involving experimental animals were performed in accordance with protocols approved by the Committee for Animal Research of Xiamen University (Xiamen, China) and the Guide for the Care and Use of Laboratory Animals (National Institutes of Health publication no. 86-23, revised 1985).

Cell cultures and reagents. HepG2 and $\mathrm{HuH} 7$ cells were obtained from the American Type Culture Collection (Manassas, VA, USA) and cultured in Dulbecco's modified Eagle's medium supplemented with $10 \%$ fetal bovine serum, $100 \mathrm{U} / \mathrm{ml}$ penicillin, $100 \mathrm{mg} / \mathrm{ml}$ streptomycin, $2 \mathrm{mM}$ glutamine, $1 \mathrm{mM}$ sodium pyruvate and $0.1 \mathrm{mM}$ non-essential amino acids at $37^{\circ} \mathrm{C}$ with $5 \% \mathrm{CO}_{2}$ (All from Gibco Life Technologies, Carlsbad, CA, USA).

Construction of ECHS1-interfering vectors. A plasmid encoding a short interfering RNA (siRNA) targeted to ECHS1 was constructed as described previously (13). Briefly, two siRNA target sequences (5'-GCCCATATCGTTTCATAGCTT-3' and 5'-GTAGATGAGATGTGACGAATT-3') for the ECHS1 gene (siECHS1-1 and siECHS1-2) were selected using the RNAi Target Selector algorithm (http://www.clontech.com/ GB/Support/Online_Tools), and the most efficient of the two (siECHS1-2) was selected for use using the Basic Local Alignment Search Tool (http://blast.ncbi.nlm.nih.gov/Blast. cgi) in the present study. The oligonucleotides were annealed, to form siRNA duplexes, and were cloned into the BbsI and $\mathrm{XbaI}$ restriction sites of the pu6 vector (Dharmacon, Inc. Chicago, IL, USA) and named siECHS1-1 and siECHS1-2. The final clones were verified by DNA sequencing (Beijing Genomics Institution, Beijing, China).

Establishment of stable ECHS1-knockdown cell pools. HepG2 and $\mathrm{HuH7}$ cells were transfected with pu6-siECHS1 using Lipofectamine ${ }^{\circledR} 2000$ reagent (Invitrogen Life Technologies, Carlsbad, CA, USA). Cells transfected with empty pu6 vectors served as controls. Cells were cultured in medium containing $1 \mu \mathrm{g} / \mathrm{ml}$ puromycin (Invitrogen Life Technologies) for selection $(21,22)$. Following two weeks of culture, ECHS1 protein expression was examined by western blotting (as described in the 'Western blot analysis' section) to validate knockdown efficiency; and these experiments were repeated three times. The ECHS1-knockdown cell lines were named HepG2-siECHS1 and HuH7-siECHS1 and the control cell lines were named HepG2-pu6 and HuH7-pu6.

Cell proliferation assays. Cell proliferation was measured by Cell Counting kit-8 (CCK-8) assay (Dojindo Molecular
Technologies, Inc., Kumamoto, Japan). Cells were cultured at a density of $\sim 2,500$ cells/well in a 96-well plate, in which $10 \mu \mathrm{l}$ CCK-8 was added to $100 \mu \mathrm{l}$ culture medium. The cells were incubated for $1.5 \mathrm{~h}$ at $37^{\circ} \mathrm{C}$ and absorbance was measured at $450 \mathrm{~nm}$ using a Fluorescence Multi-Well Plate Reader CytoFluor 4000-2 (PerSeptive Bio-systems, Inc., Framingham, MA, USA). Experiments were repeated three times.

The 5-bromo-2-deoxyuridine (BrdU) assay was performed with the BrdU Cell Proliferation Assay kit according to the manufacturer's instructions (Cell Signaling Technology, Inc., Danvers, MA, USA). Briefly, cells were seeded into three wells of a 96 -well plate at $0.5 \times 10^{4}, 1 \times 10^{4}$ and $2 \times 10^{4}$ cells/well and incubated at room temperature for $24 \mathrm{~h}$. BrdU (10 $\mu \mathrm{l})$ was added to each well and incubated at room temperature for an additional $4 \mathrm{~h}$. Following removal of the medium, $100 \mu \mathrm{l} /$ well Fixing/Denaturing solution was added and incubated at room temperature for $30 \mathrm{~min}$, followed by antibody detection solution for $1 \mathrm{~h}$. The plate was washed three times and the anti-mouse horseradish peroxidase-conjugated secondary immunoglobulin $\mathrm{G}(\mathrm{IgG})$ antibody was added and incubated for $30 \mathrm{~min}$, followed by $100 \mu \mathrm{TMB}$ Substrate for $30 \mathrm{~min}$. The reaction was terminated with STOP solution and absorbance was measured at $450 \mathrm{~nm}$ on the PerSeptive Bio-systems, Inc. microplate reader.

Enzymatic activity of ECHS1. ECHS1 activity was assayed according to the manufacturer's instructions of the Cellular Short Chain ENOYL COA HYDRATASE Activity Assay kit (Genmed Scientifics, Inc., Arlington, MA, USA). Activity was measured by determining absorbance at $263 \mathrm{~nm}$ with the PerSeptive Bio-systems, Inc. microplate reader. Protein concentrations were determined by the Bradford method (23) with Quick Start Bradford Protein Assay kit 1 assay solution and bovine serum albumin (BSA) was used as the standard (Bio-Rad Laboratories, Inc., Hercules, CA, USA).

Xenograft assay. To determine the oncogenicity of ECHS1, ten 4-6 month-old male nude mice were randomly divided into two groups and administered $6 \times 10^{6} \mathrm{HepG} 2$-siECHS1 or HepG2-pu6 cells/mouse via injection to the gluteal region. HepG2 cells were selected for use, due to Huh7 cells exhibiting poor tumorigenesis effects in preliminary experiements. Tumor size was assessed every three days using calipers and the tumor volume was calculated using the formula: [length $(\mathrm{mm}) \mathrm{x}$ width $\left.(\mathrm{mm})^{2}\right] / 2$.

Western blot analysis. The total protein was extracted from cells and tissue specimens using Mammalian CellLysis reagent (Fermentas; Thermo Fisher Scientific, Pittsburgh, PA, USA) according to the manufacturer's instructions. The proteins were resolved using 10, 12 or 15\% SDS-PAGE and detected with the appropriate antibodies. The following primary antibodies were all purchased from Cell Signaling Technology, Inc. (Danvers, MA, USA), apart from those against ECHS1 and EGFR which were purchased from ProteinTech Group, Inc. (Chicago, IL, USA): Mouse monoclonal proliferating cell nuclear antigen (PCNA; 1:1,000; \#2586), rabbit monoclonal AKT (1:1,000; \#4691), rabbit monoclonal phosphorylated (p-)Akt(Ser473) (1:1,000; \#4060), ERK1/2 (1:1,000; \#4695), 
A

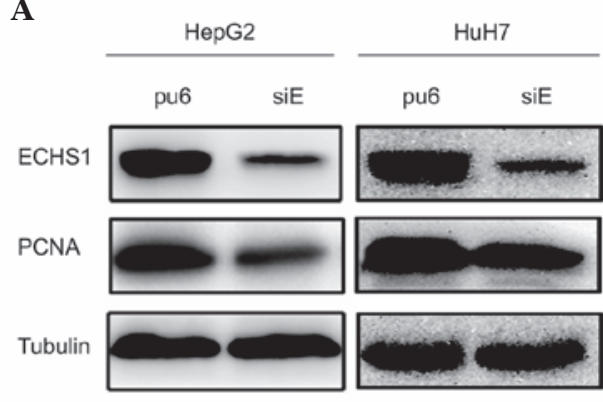

CD $\longrightarrow$ pu6
$(450 \mathrm{~nm})-\mathrm{SiECHS} 1$

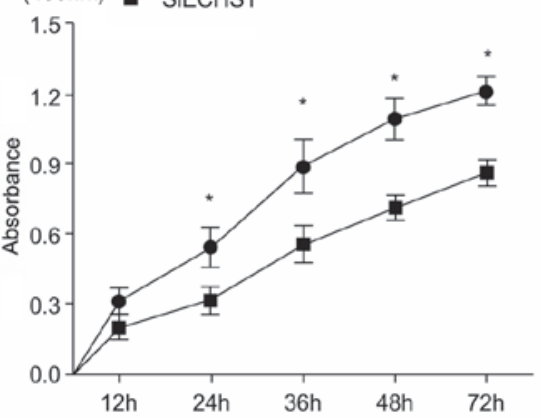

$\mathbf{E}$

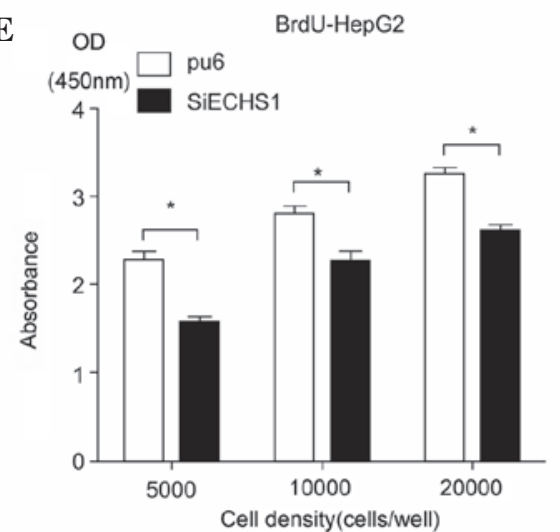

B

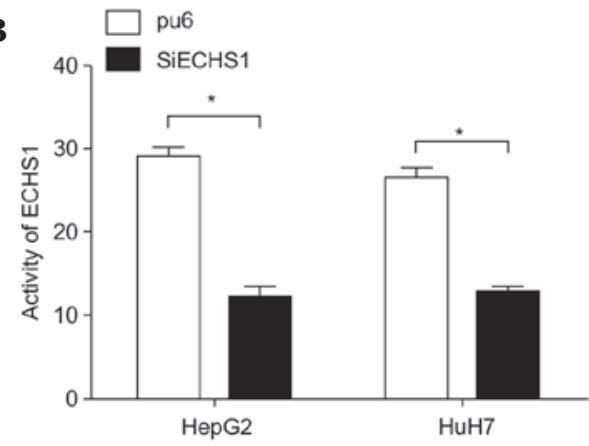

D

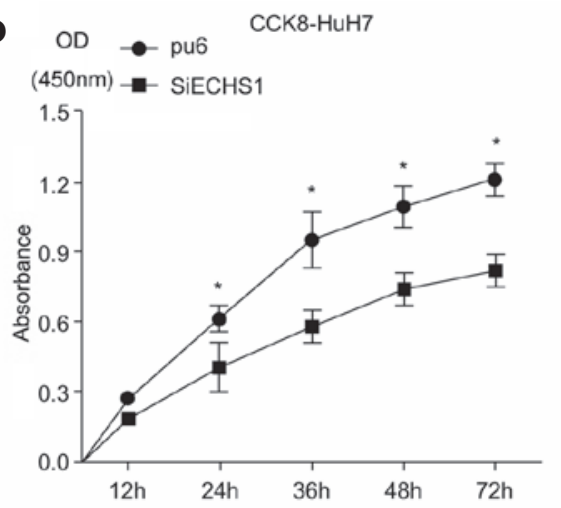

F

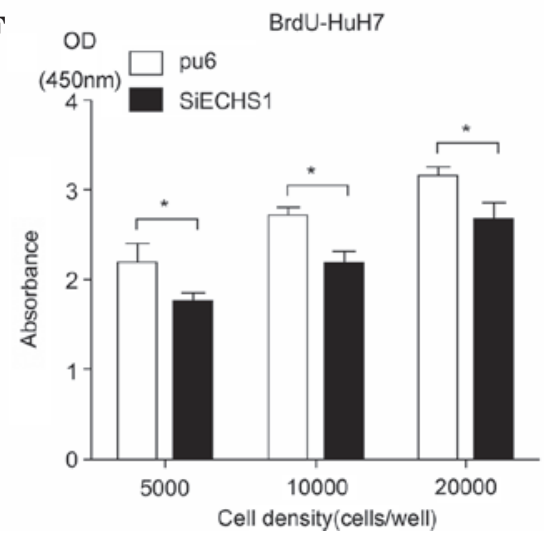

Figure 1. Silencing of ECHS1 attenuates HCC cell proliferation in vitro. (A) Western blot analysis confirmed siRNA silencing of ECHS1 and proliferation marker PCNA expression. Cells transfected with empty vectors were used as controls and tubulin was used as an internal control. (B) Relative enzymatic activity of ECHS1 in HepG2-siECHS1, Huh7-siECHS1 and control cells, "P<0.01. (C and D) CCK8 assay of cell proliferation in HepG2-siECHS1, Huh7-siECHS1 and control cells, performed every $12 \mathrm{~h}$ for $72 \mathrm{~h} ;{ }^{*} \mathrm{P}<0.05$. (E and F) BrdU assay of cell proliferation in HepG2-siECHS1, Huh7-siECHS1 and control cells following $24 \mathrm{~h}$ incubation at $0.5 \times 10^{4}, 1 \times 10^{4}$ and $2 \times 10^{4}$ cells/well, respectively; ${ }^{*} \mathrm{P}<0.05$. ESCHS1, enoyl-coenzyme A hydratase short chain $1 ;$ HCC, hepatocellular carcinoma; siRNA, short interfering RNA; PCNA, proliferating cell nuclear antigen; CCK8, cell counting kit-8; BrdU, 5-bromo-2-deoxyuridine; pu6, control cells; siE, ECHS1 siRNA-transfected cells; SiECHS1, ESCHS1 siRNA-transfected cells.

rabbit monoclonal p-ERK1/2(Thr202/Tyr204) (1:1,000; \#4370), rabbit polyclonal nuclear factor (NF)- $\mathrm{B}(1: 1,000$; \#4882), rabbit polyclonal p-NF- $\mathrm{BB}(1: 1,000$; \#4810), rabbit monoclonal p-EGFR(Tyr1068) (1:1,000; \#11862), rabbit monoclonal glycogen synthase kinase (GSK)-3 $\beta$ (1:1,000; \#12456), rabbit monoclonal p-GSK-3 $\beta$ (Ser9) (1:1,000; \#5558), rabbit monoclonal C-Myc (1:1,000; \#13987), rabbit monoclonal $\beta$-catenin $(1: 1,000 ; \# 8480)$, rabbit monoclonal cyclin dependent kinase (CDK)4 (1:1,000; \#12790), rabbit monoclonal CDK6 (1:1,000; \#13331), rabbit monoclonal cyclin D1 (1:1,000; \#2978), mouse monoclonal cyclin D3 (1:1,000; \#2936), rabbit polyclonal p15 (1:1,000; \#4822), rabbit polyclonal p16 (1:1,000; \#4824), mouse monoclonal p21 (1:1,000; \#2946), rabbit monoclonal p27 (1:1,000; \#3686), rabbit polyclonal ECHS1 $(1: 2,000 ; 11305-1-A P)$ and rabbit polyclonal
EGFR (1:3,000; 18986-1-AP). The antibodies were diluted in 5\% w/v BSA, $1 \mathrm{X}$ Tris-buffered saline and $0.1 \%$ Tween-20 (Amresco, Solon, OH, USA) at $4^{\circ} \mathrm{C}$ with gentle shaking, overnight. Subsequently, the membranes were washed and incubated with the following secondary antibodies from Cell Signaling Technology, Inc.: Anti-mouse IgG (1:2,500; \#7076) and anti-rabbit IgG (1:2500; \#7074) conjugated to horseradish peroxidase.

Immunoreactivity was detected with an Enhanced Chemiluminescence kit (GE Healthcare Life Sciences, Chalfont, UK) and quantified using densitometry with the ImageQuant LAS 4000 mini system (GE Healthcare Life Sciences). Tubulin (Cell Signaling Technology, Inc.) was used as a loading control. 
A

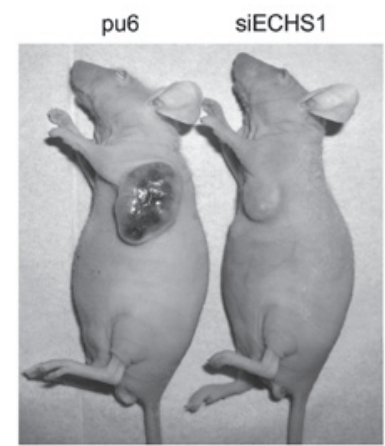

$\mathbf{C}$

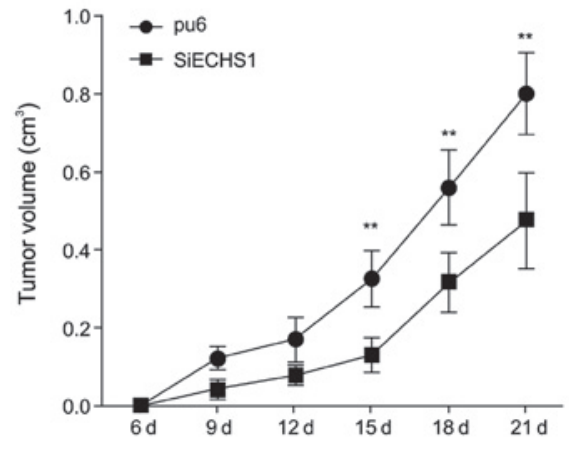

B

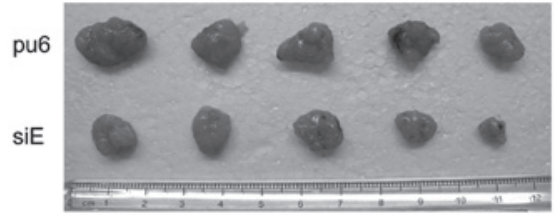

D

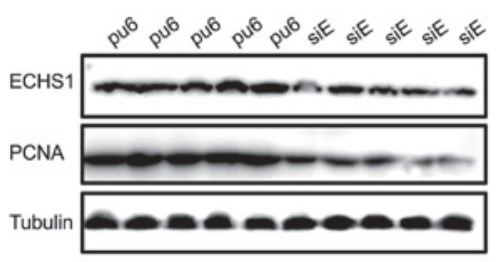

$\mathbf{E}$

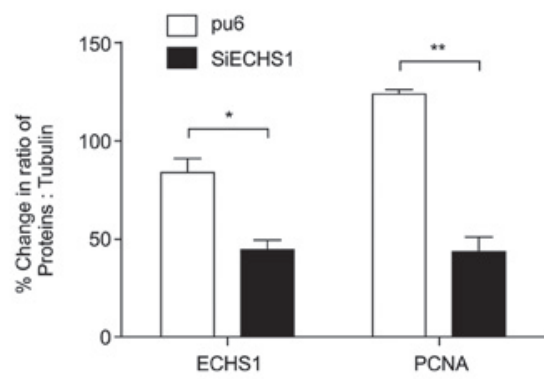

Figure 2. ECHS1 silencing inhibits the growth of xenograft tumors in vivo. Nude mice were subcutaneously injected with HepG2-siECHS1 or HepG2-pu6 cells, and the tumors were excised 21 days later. (A and B) Representative images of the tumors in the nude mice and following excision. (C) Xenograft tumor growth was monitored every 3 days for 21 days; values are expressed as the mean tumor volume \pm standard deviation ( $\mathrm{n}=5$ mice per group; $\left.{ }^{* *} \mathrm{P}<0.01\right)$. (D) ECHS1 knockdown and decreased PCNA levels were maintained in vivo. (E) Bands were quantified by optical density scanning; the percent changes in the ratio of the indicated proteins to tubulin are presented as the mean \pm standard deviation $\left({ }^{*} \mathrm{P}<0.05,{ }^{* *} \mathrm{P}<0.01\right)$. ESCHS1, enoyl-coenzyme A hydratase short chain 1; HCC, hepatocellular carcinoma; siRNA, short interfering RNA; PCNA, proliferating cell nuclear antigen; pu6, control cells; siE, ECHS1 siRNA-transfected cells; SiECHS1, ESCHS1 siRNA-transfected cells.

Statistical analysis. Experimental data are presented as the mean \pm standard deviation of at least three independent experiments, as calculated using SPSS, version 13.0 (SPSS, Inc., Chicago, IL, USA). The independent-samples and paired-samples t-tests were used to compare data. $\mathrm{P}<0.05$ was considered to indicate a statistically significant difference. Statistical graphs with error bars representing the standard deviation of the mean were created using GraphPad Prism 5 Software (GraphPad Software, Inc., San Diego, CA, USA).

\section{Results}

Establishment of stable ECHS1-knockdown cell pools using siRNA in human HepG2 and HuH7 cells. HepG2 and $\mathrm{HuH7}$ cells were transfected with ECHS1 siRNA or the empty pu6 vector (control) to stably establish HepG2-siECHS1 and HuH7-siECHS1 ECSH1 knocked-down cell pools and HepG2-pu6 and HuH7-pu6 control cells. Western blot analysis revealed that ECHS1 expression was downregulated in the HepG2-siECHS1 and HuH7-siECHS1 cells compared with that of the HepG2-pu6 and HuH7-pu6 cells, respectively (Fig. 1A). As expected, the enzymatic activity of ECHS1 was attenuated in the HepG2-siECHS1 and HuH7-siECHS1 cells compared with that of HepG2-pu6 and HuH7-pu6 cells (Fig. 1B).

Silencing of ECHS1 attenuates HCC cell proliferation in vitro. A reduction in the proliferation rate of HepG2-siECHS1 and HuH7-siECHS1 cells compared with that of the control cells was identified by CCK8 assay following 24, 36, 48 and $72 \mathrm{~h}$ of incubation (Fig. 1C and D). The BrdU staining results at $0.5 \times 10^{4}, 1 \times 10^{4}$ and $2 \times 10^{4}$ cells/well following $24 \mathrm{~h}$ of incubation were consistent with those of the CCK-8 assay (Fig. 1E and F). PCNA, a proliferation marker, was also downregulated in HepG2-siECHS1 and HuH7-siECHS1 cells (Fig. 1A). Therefore, silencing of ECHS1 inhibited the proliferation of HepG2 and HuH7 cells. 
A

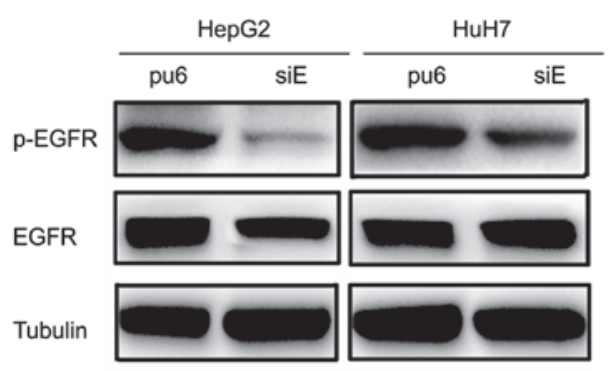

C

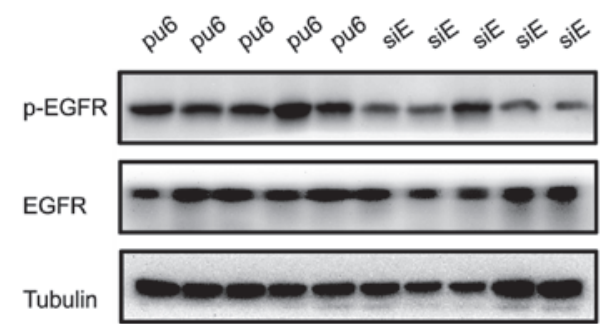

E

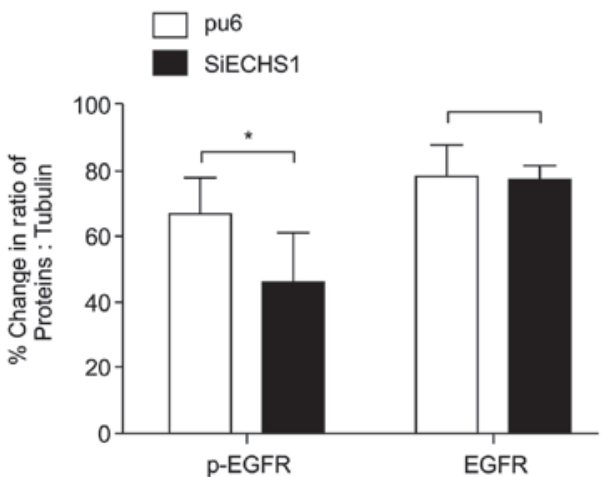

B

$\frac{\text { HepG2 }}{\text { pu6 siE }} \stackrel{\text { HuH7 }}{\text { pu6 siE }}$

p-AKT

AKT

p-ERK

ERK
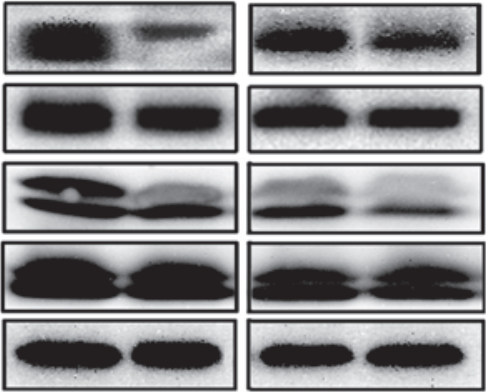

Tubulin

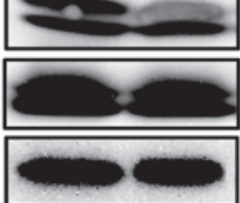

D

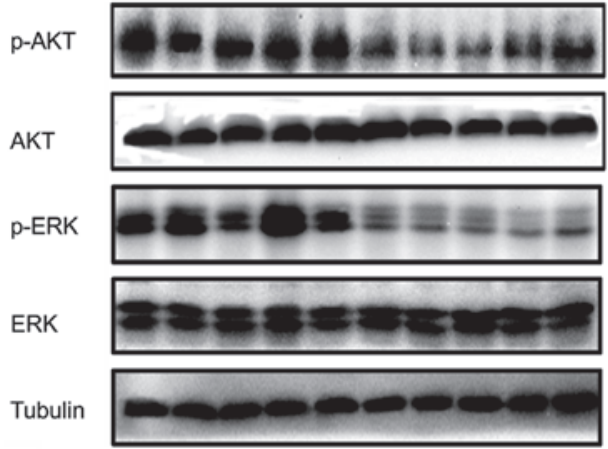

F

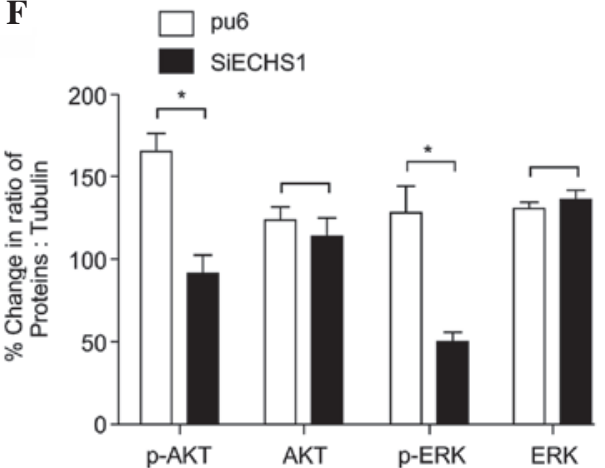

Figure 3. Silencing of ECHS1 downregulates hepatocellular carcinoma cell proliferation by inhibiting EGFR signaling in vitro and vivo. Western blot analysis of EGFR, AKT, and ERK phosphorylation in (A and B) HepG2- and Huh7-siECHS1 and control cells and (C and D) tumors derived from ECHS1 knock-down cells and controls. Bands were quantified by optical density scanning of (E) EGFR, (F) AKT and ERK in vivo (as indicated in C and D). The percent changes in the ratio of the indicated proteins to tubulin are presented as the mean \pm standard deviation; ${ }^{*} \mathrm{P}<0.05,{ }^{* * *} \mathrm{P}<0.01$. ESCHS1, enoyl-coenzyme A hydratase short chain 1; siRNA, short interfering RNA; pu6, control cells; siE, ECHS1 siRNA-transfected cells; SiECHS1, ESCHS1 siRNA-transfected cells; EGFR, epidermal growth factor receptor; ERK, extracellular signal-regulated kinase; p-, phosphorylated.

Silencing of ECHS1 suppresses xenograft tumor growth. ECHS1 knockdown suppressed HCC cell proliferation in vitro; therefore, the role of ECHS1 in HCC development in vivo was subsequently evaluated. Nude mice were subcutaneously injected with HepG2-siECHS1 and HepG2-pu6 cells to induce xenograft tumors (Fig. 2A and B), which developed over time (Fig. 2C). Slower growth was observed in tumors derived from HepG2-siECHS1 cells than in those derived from HepG2-pu6 cells (Fig. 2A-C). Western blotting verified that ECHS1 knockdown was maintained in the transplanted tumors, and PCNA expression remained lower in mice injected with HepG2-siECHS1 cells than those of the controls (Fig. 2D and E). Therefore it was concluded that the absence of ECHS1 was responsible for the inhibition of tumor growth.
ECHS1 regulates $H C C$ cell proliferation via the EGFR signaling pathway. To define the signaling pathways involved in ECHS1-mediated regulation of HCC cell proliferation, western blotting was used to detect the phosphorylation levels of EGFR and its downstream effectors ERK and AKT, which mediate proliferation, survival, apoptosis and cell cycle progression. The expression levels of p-EGFR, p-ERK1/2 (Thr202/Tyr204) and p-AKT (Ser473) were markedly decreased in HepG2-siECHS1 and $\mathrm{HuH} 7$-siECHS1 cells compared with those of the control cells (Fig. 3A and B). EGFR, ERK1/2 and AKT phosphorylation were also analyzed in xenograft tumors derived from ECHS1 knock-down cells. Consistent with the results of the in vitro study, the phosphorylation of EGFR, ERK1/2 and AKT was downregulated in ECHS1 knock-down tumor tissues at the end of the study period (Fig. 3C-F). The expression of GSK-3 $\beta$, which is a 
A
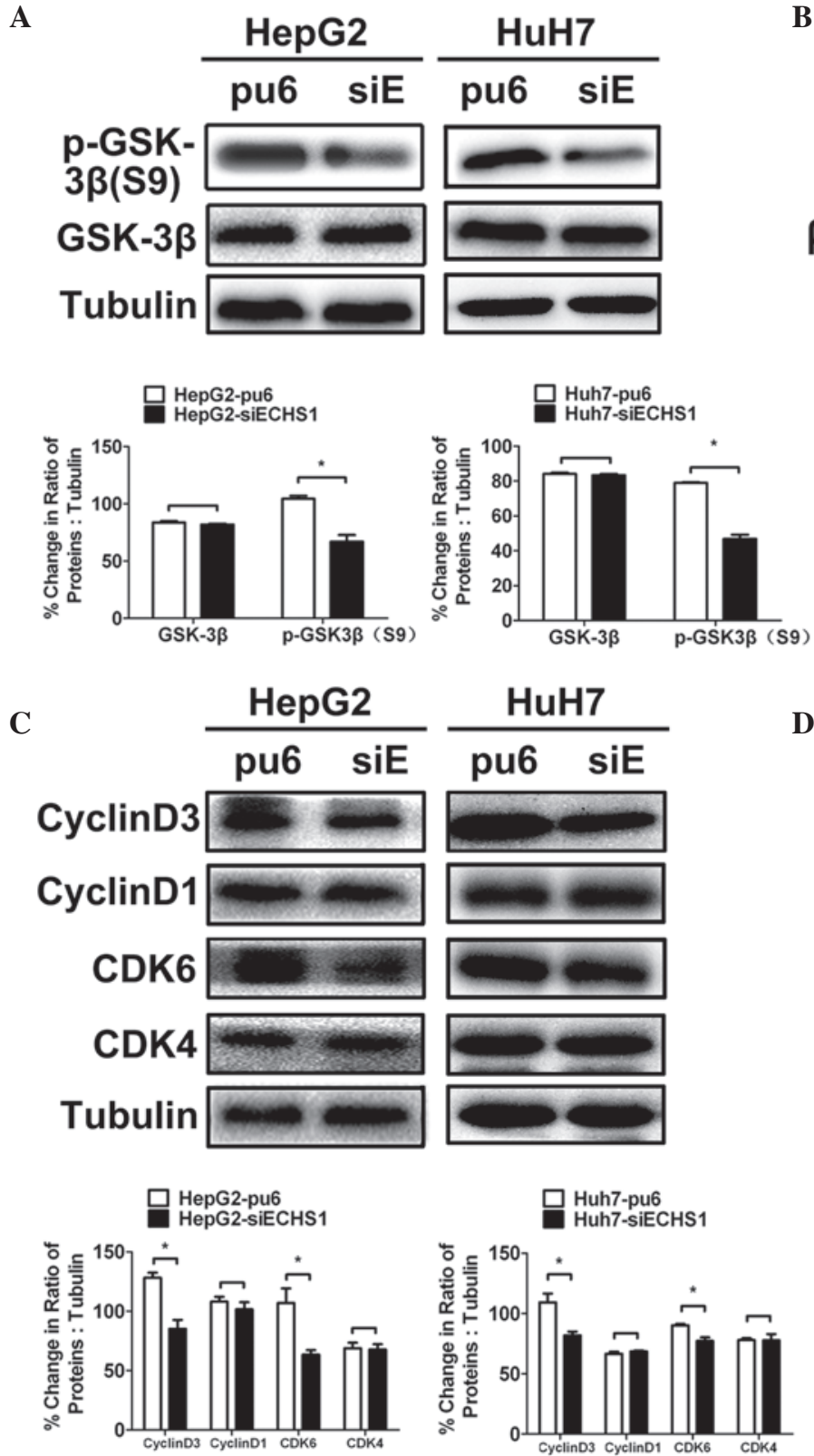
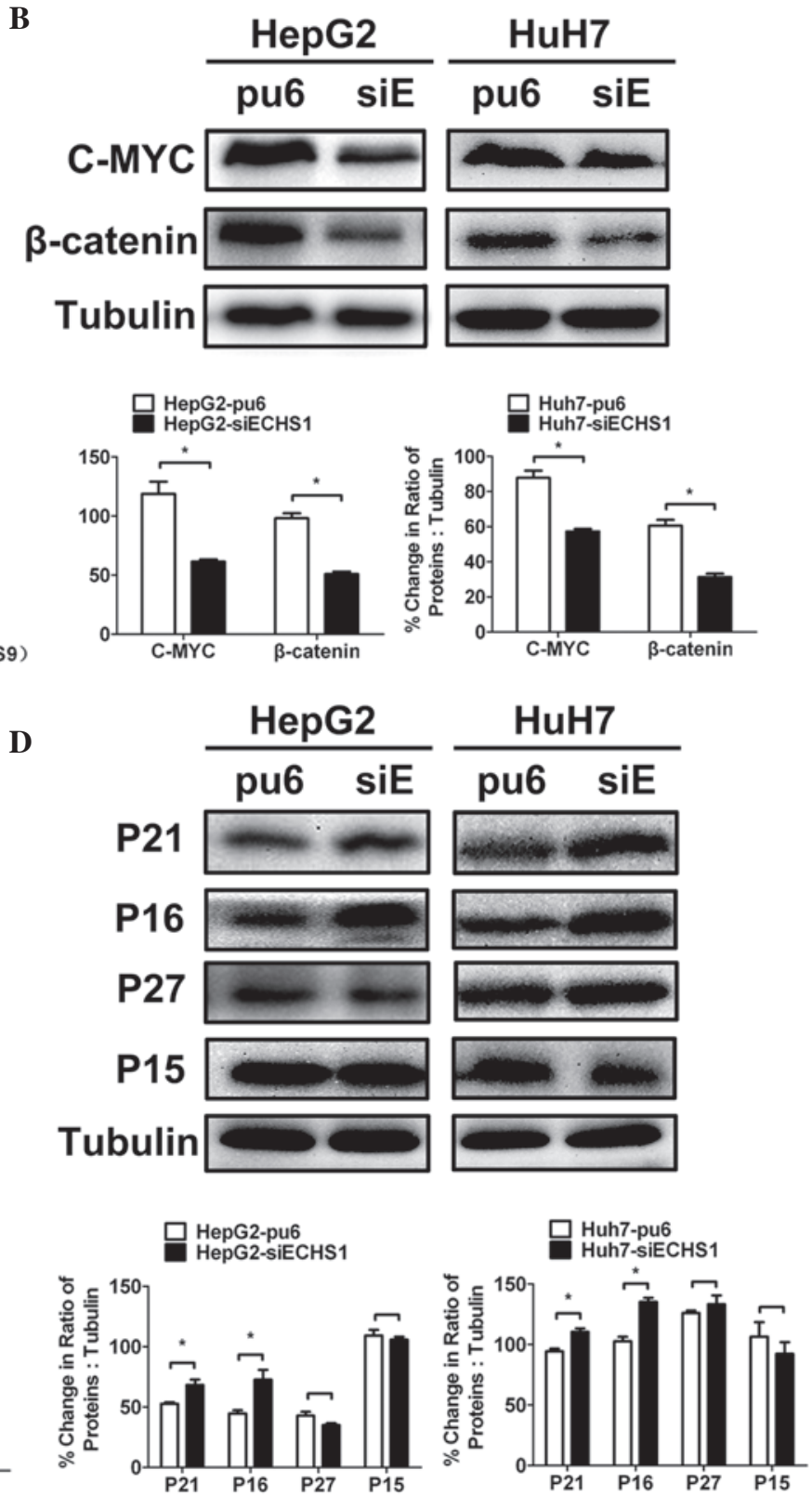

Figure 4. Silencing of ECHS1 alters the expression of proteins involved in Wnt/ $\beta$-catenin signaling and cell cycle regulation. (A and B) Western blot analysis and quantification of GSK-3 $\beta$, p-GSK-3 $\beta$ (Ser9), $\beta$-catenin and C-MYC in HepG2- and Huh7-siECHS1 and control cells. (C and D) Western blot analysis and quantification of cell cycle-associated proteins in HepG2-siECHS1, Huh7-siECHS1 and control cells. Data are presented as the mean \pm standard deviation ("P $<0.05$, ${ }^{* *} \mathrm{P}<0.01$ vs. control). ESCHS1, enoyl-coenzyme A hydratase short chain 1; siRNA, short interfering RNA; pu6, control cells; siE, ECHS1 siRNA-transfected

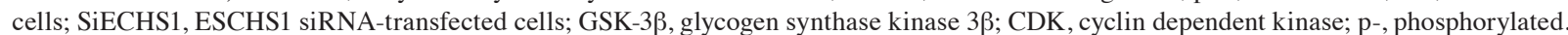

major inhibitor of the Wnt $/ \beta$-catenin pathway, was also assessed. Phosphorylation of GSK-3 $\beta$ at serine-9 was markedly reduced in HepG2-siECHS1 and Huh7-siECHS1 cells compared with that of the control cells (Fig. 4A), thereby increasing the negative regulatory effect of GSK-3 $\beta$ on the $\mathrm{Wnt} / \beta$-catenin pathway. Concurrently, C-Myc and $\beta$-catenin expression were also downregulated in HepG2- and HuH7-siECHS1 cells (Fig. 4B). However, no significant differences in NF- $\kappa \mathrm{B}$ phosphorylation were observed in these cells (data not shown). Therefore, it was hypothesized that ECHS1 may regulate cancer cell proliferation in vitro and in vivo via an EGFR-mediated signaling pathway.

ECHS1 modulates the expression of cell cycle regulators. The expression levels of various cell cycle regulators that are directly associated with cellular proliferation were evaluated. The expression of cyclin D3 and CDK6 was reduced in HepG2- and Huh7-siECHS1 cells (Fig. 4C), whereas expression of the CDK inhibitors p16 and p21 was enhanced (Fig. 4D). Expression levels of CDK4, cyclin D1, p15 and p27 were not significantly altered in HepG2- and Huh7-siECHS1 cells compared with those of the control cells (Fig. 4C and D). It was therefore confirmed that ECHS1 knockdown in HCC cells suppressed cyclin D3 and CDK6 expression and enhanced the expression of p16 and p21.

\section{Discussion}

Late diagnosis and high recurrence are the leading causes of poor survival amongst patients with HCC (24). Therefore, 
elucidation of the molecular mechanisms that mediate HCC progression and the identification of efficient therapeutic targets are urgently required. A previous study by our group reported that ECHS1 binds HBs to enhance HepG2 cell apoptosis. It was also demonstrated that knockdown of endogenous ECHS1 attenuated the cell growth, proliferation and migration of HepG2 cells. Furthermore, the involvement of pro-apoptotic and pro-survival proteins and Akt phosphorylation levels in HepG2 cells was defined, suggesting that ECHS1 is a significant regulator of HCC progression (13). Zhu et al (25) confirmed that ECHS1 knockdown inhibited HCC cell proliferation via suppression of AKT activity in HepG2 cells. However, to the best of our knowledge, the upstream and downstream regulators of this signaling cascade have not previously been investigated, and the mechanisms underlying the involvement of ECHS1 in HCC proliferation have remained elusive.

In the present study, the high expression levels of ECHS1 in human HepG2 and Huh-7 cells were verified. Subsequently, the suppression of proliferation in HepG2 and Huh-7 cells by stable ECHS1 knockdown was observed. Tumors derived from transplanted HepG2-siECHS1 cells grew more slowly than tumors derived from HepG2-pu6 cells. The downregulation of proliferation marker PCNA (26) in ECHS1 knockdown cells was also observed in vitro and in vivo. These results suggested that ECHS1 mediates HCC proliferation; however, elucidation of the mechanisms underlying this process require further investigation.

TheEGFR-induced mitogenic signaling pathway is activated in various malignancies, including HCC (14-17). EGFR phosphorylation leads to activation of the PI3K/Akt and MEK/ERK signaling cascades, which are involved in cell proliferation, survival, motility, differentiation and angiogenesis (27-29). EGFR induces activation of the Ras/Raf/MEK/ERK pathway via Grb2 or Shc adaptor proteins, and the PI3K/AKT/mTOR pathway via recruitment of the $\mathrm{p} 85$ regulatory subunit to the activated receptors (30). In the present study, EGFR phosphorylation levels were reduced in ECHS1 knock-down HepG2 and Huh7 cells; and the expression of downstream effectors p-AKT and p-ERK were also reduced in vitro and in xenograft tumors. p-ERK1/2 and p-Akt(ser473) are highly expressed in HCC tissues, and activation of the ERK and AKT pathways is indicative of poor prognosis in patients with HCC (31). Certain studies have focused on evaluating the synergy between the PI3K/Akt and MEK/ERK pathways in $\operatorname{HCC}(31,32)$. It was therefore hypothesized that ECHS1 may function via PI3K/Akt and MEK/ERK signaling to mediate liver tumor development in an EGFR-dependent manner.

Akt-mediated phosphorylation of GSK-3 $\beta$ also influences the Wnt/ $\beta$-catenin pathway and the epithelial mesenchymal transition (33). As expected, serine-9 phosphorylation of GSK-3 $\beta$ was reduced in HepG2- and Huh7-siECHS1 cells compared with that of the controls, which may thereby increase the negative effect of GSK-3 $\beta$ on the Wnt $/ \beta$-catenin pathway, leading to downregulation of $\beta$-catenin. Upon activation of the Wnt pathway, $\beta$-catenin forms a complex with B-cell lymphoma-9, Pygo, plakoglobulin and T-cell factor/lymphoid enhancing factor, which results in the transcription of critical genes including cyclin D1, c-Myc, SALL4 and PPARס (33). In the present study, $\beta$-catenin, $c-M y c$ and cyclin D3 expression were decreased in response to $\mathrm{p}-\mathrm{GSK} 3 \beta$ downregulation in
ECHS1-knockdown HepG2 and Huh7 cells. It was therefore hypothesized that ECHS1 may regulate the PI3K/Akt and MEK/ERK pathways by targeting EGFR, leading to activation of the downstream signaling cascade and promoting $\mathrm{HCC}$ progression. However, the specific mechanisms underlying this function require further investigation.

Previous studies have demonstrated that EGFR regulates the cell cycle via the PI3K/Akt and MEK/ERK signaling pathways $(34,35)$. Akt regulates cell cycle progression by inducing GSK-3 $\beta$ inhibition (36), cyclin D1 degradation and p21 upregulation (37). Cell cycle regulators were therefore evaluated and it was revealed that knockdown of ECHS1 suppressed cyclin D-CDK4/6 complex formation and enhanced expression of the negative upstream regulators p16 and p21.

In conclusion, ECHS1 promoted the development of human HCC by regulating cell proliferation and cell cycle progression. Consistent with a previous study by our group, which indicated that ECHS1 may be involved in the progression of HCC (13), the results of the present study revealed a mechanistic link between ECHS1 and EGFR signal activation through PI3K/Akt and c-Raf/MEK/ERK. Moreover, ECHS1 modulated the Wnt/ $\beta$-catenin pathway through p-GSK-3 $\beta($ ser9) and the expression of cell cycle markers, including cyclin D1/3, CDK4/6, p16 and p21. These results provide an insight into the critical role of ECHS1 in the intricate EGFR signal transduction network, which may result in the development of novel interventions using EGFR-targeted therapies for the treatment of HCC. However, the precise mechanism by which ECHS1 regulates EGFR requires further evaluation.

\section{Acknowledgements}

The present study was supported by grants from the National Key Basic Research Program of China (no. 2013CB933904), National Key Sci-Tech Special Project of China (no. 2012ZX10002-011-005), Projects of Xiamen Science and Technology Program (no. 3502Z20130030) and the National Natural Science Foundation of China (no. 81171976).

\section{References}

1. Parkin DM, Bray F, Ferlay J and Pisani P: Global cancer statistics, 2002. CA Cancer J Clin 55: 74-108, 2005.

2. Tsim NC, Frampton AE, Habib NA and Jiao LR: Surgical treatment for liver cancer. World J Gastroenterol 16: 927-933, 2010.

3. Guégan JP, Ezan F, Théret N, Langouët S and Baffet G: MAPK signaling in cisplatin-induced death: predominant role of ERK1 over ERK2 in human hepatocellular carcinoma cells. Carcinogenesis 34: 38-47, 2013.

4. Tsao CM, Yan MD, Shih YL, et al: SOX1 functions as a tumor suppressor by antagonizing the $\mathrm{WNT} / \beta$-catenin signaling pathway in hepatocellular carcinoma. Hepatology 56: 2277-2287, 2012.

5. Yang J, Cai X, Lu W, Hu C, Xu X, Yu Q and Cao P: Evodiamine inhibits STAT3 signaling by inducing phosphatase shatterproof 1 in hepatocellular carcinoma cells. Cancer Lett 328: 243-251, 2013.

6. Yang P, Li QJ, Feng Y, et al: TGF- $\beta$-miR-34a-CCL22 signaling-induced Treg cell recruitment promotes venous metastases of HBV-positive hepatocellular carcinoma. Cancer Cell 22: 291-303, 2012.

7. Agnihotri G and Liu HW: Enoyl-CoA hydratase. reaction, mechanism, and inhibition. Bioorg Med Chem 11: 9-20, 2003.

8. Lin JF, Xu J, Tian HY, et al: Identification of candidate prostate cancer biomarkers in prostate needle biopsy specimens using proteomic analysis. Int J Cancer 121: 2596-2605, 2007. 
9. Komori T, Takemasa I, Higuchi H, et al: Identification of differentially expressed genes involved in colorectal carcinogenesis using a cDNA microarray. J Exp Clin Cancer Res 23: 521-527, 2004.

10. Yeh CS, Wang JY, Cheng TL, Juan CH, Wu CH and Lin SR: Fatty acid metabolism pathway play an important role in carcinogenesis of human colorectal cancers by Microarray-Bioinformatics analysis. Cancer Lett 233: 297-308, 2006.

11. Liu X, Feng R and Du L: The role of enoyl-CoA hydratase short chain 1 and peroxiredoxin 3 in PP2-induced apoptosis in human breast cancer MCF-7 cells. FEBS Lett 584: 3185-3192, 2010.

12. Chang Y, Wang SX, Wang YB, et al: ECHS1 interacts with STAT3 and negatively regulates STAT3 signaling. FEBS Lett 587: 607-613, 2013.

13. Xiao CX, Yang XN, Huang QW, et al: ECHS1 acts as a nove HBsAg-binding protein enhancing apoptosis through the mitochondrial pathway in HepG2 cells. Cancer Lett 330: 67-73, 2013

14. Kira S, Nakanishi T, Suemori S, Kitamoto M, Watanabe Y and Kajiyama G: Expression of transforming growth factor alpha and epidermal growth factor receptor in human hepatocellular carcinoma. Liver 17: 177-182, 1997.

15. Ito Y, Takeda T, Sakon M, et al: Expression and clinical significance of erb-B receptor family in hepatocellular carcinoma. Br J Cancer 84: 1377-1383, 2001.

16. Prigent SA and Lemoine NR: The type 1 (EGFR-related) family of growth factor receptors and their ligands. Prog Growth Factor Res 4: 1-24, 1992.

17. Barnard JA, Beauchamp RD, Russell WE, Dubois RN and Coffey RJ: Epidermal growth factor-related peptides and their relevance to gastrointestinal pathophysiology. Gastroenterology 108: 564-580, 1995.

18. DeCicco LA, Kong J and Ringer DP: Carcinogen-induced alteration in liver epidermal growth factor receptor distribution during the promotion stage of hepatocarcinogenesis in rat. Cancer Lett 111: 149-156, 1997.

19. Aravalli RN, Steer CJ and Cressman EN: Molecular mechanisms of hepatocellular carcinoma. Hepatology 48: 2047-2063, 2008.

20. Whittaker S, Marais R and Zhu AX: The role of signaling pathways in the development and treatment of hepatocellular carcinoma. Oncogene 29: 4989-5005, 2010.

21. Jazag A, Ijichi H, Kanai F, et al: Smad4 silencing in pancreatic cancer cell lines using stable RNA interference and gene expression profiles induced by transforming growth factor-beta. Oncogene 24: 662-671, 2005

22. Guleng B, Tateishi K, Ohta M, et al: Blockade of the stromal cell-derived factor-1/CXCR4 axis attenuates in vivo tumor growth by inhibiting angiogenesis in a vascular endothelial growth factor-independent manner. Cancer Res 65: 5864-5871, 2005.

23. Bradford MM: A rapid and sensitive method for the quantitation of microgram quantities of protein utilizing the principle of protein-dye binding. Anal Biochem 72: 248-254, 1976.
24. Llovet JM, Burroughs A and Bruix J: Hepatocellular carcinoma. Lancet 362: 1907-1917, 2003.

25. Zhu XS, Dai YC, Chen ZX, Xie JP, Zeng W, Lin YY and Tan QH: Knockdown of ECHS1 protein expression inhibits hepatocellular carcinoma cell proliferation via suppression of Akt activity. Crit Rev Eukaryot Gene Expr 23: 275-282, 2013.

26. Kelman $Z$ and O'Donnell M: Structural and functional similarities of prokaryotic and eukaryotic DNA polymerase sliding clamps. Nucleic Acids Res 23: 3613-3620, 1995.

27. Carpenter G: Receptors for epidermal growth factor and other polypeptide mitogens. Annu Rev Biochem 56: 881-914, 1987.

28. Schlessinger J: Cell signaling by receptor tyrosine kinases. Cell 103: 211-225, 2000.

29. Yarden Y and Sliwkowski MX: Untangling the ErbB signalling network. Nat Rev Mol Cell Biol 2: 127-137, 2001.

30. Soltoff SP, Carraway KR III, Prigent SA, Gullick WG and Cantley LC: ErbB3 is involved in activation of phosphatidylinositol 3-kinase by epidermal growth factor. Mol Cell Biol 14: 3550-3558, 1994.

31. Schmitz KJ, Wohlschlaeger J, Lang $\mathrm{H}$, et al: Activation of the ERK and AKT signalling pathway predicts poor prognosis in hepatocellular carcinoma and ERK activation in cancer tissue is associated with hepatitis C virus infection. J Hepatol 48: 83-90, 2008.

32. Saxena NK, Sharma D, Ding X, Lin S, Marra F, Merlin D and Anania FA: Concomitant activation of the JAK/STAT, PI3K/AKT, and ERK signaling is involved in leptin-mediated promotion of invasion and migration of hepatocellular carcinoma cells. Cancer Res 67: 2497-2507, 2007.

33. Steelman LS, Chappell WH, Abrams SL, et al: Roles of the Raf/MEK/ERK and PI3K/PTEN/Akt/mTOR pathways in controlling growth and sensitivity to therapy-implications for cancer and aging. Aging (Albany NY) 3: 192-222, 2011.

34. Bill HM, Knudsen B, Moores SL, Muthuswamy SK, Rao VR, Brugge JS and Miranti CK: Epidermal growth factor receptor-dependent regulation of integrin-mediated signaling and cell cycle entry in epithelial cells. Mol Cell Biol 24: 85868599,2004

35. Lui VW and Grandis JR: EGFR-mediated cell cycle regulation. Anticancer Res 22: 1-11, 2002.

36. Horn S, Endl E, Fehse B, Weck MM, Mayr GW and Jücker M: Restoration of SHIP activity in a human leukemia cell line downregulates constitutively activated phosphatidylinositol 3-kinase/Akt/GSK-3beta signaling and leads to an increased transit time through the G1 phase of the cell cycle. Leukemia 18: 1839-1849, 2004.

37. Diehl JA, Cheng M, Roussel MF and Sherr CJ: Glycogen synthase kinase-3beta regulates cyclin D1 proteolysis and subcellular localization. Genes Dev 12: 3499-3511, 1998. 